\title{
The OmegaWhite Survey for Short-period Variable Stars. V. Discovery of an Ultracompact Hot Subdwarf Binary with a Compact Companion in a 44-minute Orbit
}

\author{
T. Kupfer ${ }^{1}$ (1) , G. Ramsay ${ }^{2}$, J. van Roestel ${ }^{3}$, J. Brooks ${ }^{4}$, S. A. MacFarlane ${ }^{3,5}$, R. Toma ${ }^{2}$, P. J. Groot ${ }^{3,5,6}$, P. A. Woudt ${ }^{5}$, L. Bildsten ${ }^{4,6}$ \\ T. R. Marsh ${ }^{7}$ (1), M. J. Green ${ }^{7}$, E. Breedt ${ }^{7}$, D. Kilkenny ${ }^{8}$, J. Freudenthal ${ }^{9}$, S. Geier ${ }^{10}$, U. Heber ${ }^{11}$ (i), S. Bagnulo ${ }^{2}$, \\ N. Blagorodnova ${ }^{1}$ (D), D. A. H. Buckley ${ }^{12}$, V. S. Dhillon ${ }^{13,14}$, S. R. Kulkarni ${ }^{1}$ (D), R. Lunnan ${ }^{1,15}$ (D), and T. A. Prince ${ }^{1}$ (D) \\ ${ }^{1}$ Division of Physics, Mathematics and Astronomy, California Institute of Technology, Pasadena, CA 91125, USA \\ ${ }^{2}$ Armagh Observatory and Planetarium, College Hill, Armagh BT61 9DG, UK \\ ${ }^{3}$ Department of Astrophysics/IMAPP, Radboud University, P.O. Box 9010, NL-6500 GL Nijmegen, The Netherlands \\ ${ }^{4}$ Department of Physics, University of California, Santa Barbara, CA 93106, USA \\ ${ }^{5}$ Department of Astronomy, University of Cape Town, Private Bag X3, Rondebosch 7700, South Africa \\ Kavli Institute for Theoretical Physics, University of California, Santa Barbara, CA 93106, USA \\ ${ }^{7}$ Department of Physics, University of Warwick, Coventry CV4 7AL, UK \\ ${ }_{9}^{8}$ Department of Physics \& Astronomy, University of the Western Cape, Private Bag X17, Bellville 7535, South Africa \\ ${ }^{9}$ Institut für Astrophysik, Georg-August-Universität Göttingen, Friedrich-Hund-Platz 1, D-37077 Göttingen, Germany \\ ${ }^{10}$ Institute for Astronomy and Astrophysics, Kepler Center for Astro and Particle Physics, Eberhard Karls University, Sand 1, D-72076 Tübingen, Germany \\ ${ }^{11}$ Dr. Remeis-Sternwarte \& ECAP, Astronomical Institute, University of Erlangen-Nuremberg, Germany \\ ${ }^{12}$ South African Astronomical Observatory, Cape Town, South Africa \\ ${ }^{13}$ Department of Physics \& Astronomy, University of Sheffield, Sheffield, S3 7RH, UK \\ ${ }^{14}$ Instituto de Astrofísica de Canarias (IAC), E-38200 La Laguna, Tenerife, Spain \\ ${ }^{15}$ The Oskar Klein Centre \& Department of Astronomy, Stockholm University, AlbaNova, SE-106 91 Stockholm, Sweden \\ Received 2017 August 11; revised 2017 October 12; accepted 2017 October 18; published 2017 December 7
}

\begin{abstract}
We report the discovery of the ultracompact hot subdwarf (sdOB) binary OW J074106.0-294811.0 with an orbital period of $P_{\text {orb }}=44.66279 \pm 1.16 \times 10^{-4}$ minutes, making it the most compact hot subdwarf binary known. Spectroscopic observations using the VLT, Gemini and Keck telescopes revealed a He-sdOB primary with an intermediate helium abundance, $T_{\text {eff }}=39400 \pm 500 \mathrm{~K}$ and $\log g=5.74 \pm 0.09$. High signal-to-noise ratio light curves show strong ellipsoidal modulation resulting in a derived sdOB mass $M_{\mathrm{sdOB}}=0.23 \pm 0.12 M_{\odot}$ with a WD companion $\left(M_{\mathrm{WD}}=0.72 \pm 0.17 M_{\odot}\right)$. The mass ratio was found to be $q=M_{\mathrm{sdOB}} / M_{\mathrm{WD}}=0.32 \pm 0.10$. The derived mass for the He-sdOB is inconsistent with the canonical mass for hot subdwarfs of $\approx 0.47 M_{\odot}$. To put constraints on the structure and evolutionary history of the sdOB star we compared the derived $T_{\text {eff }}, \log g$, and sdOB mass to evolutionary tracks of helium stars and helium white dwarfs calculated with Modules for Experiments in Stellar Astrophysics (MESA). We find that the best-fitting model is a helium white dwarf with a mass of $0.320 M_{\odot}$, which left the common envelope $\approx 1.1 \mathrm{Myr}$ ago, which is consistent with the observations. As a helium white dwarf with a massive white dwarf companion, the object will reach contact in $17.6 \mathrm{Myr}$ at an orbital period of 5 minutes. Depending on the spin-orbit synchronization timescale the object will either merge to form an $\mathrm{R} \mathrm{CrB}$ star or end up as a stably accreting AM CVn-type system with a helium white dwarf donor.
\end{abstract}

Key words: binaries (including multiple): close - stars: individual (OWJ074106.0-294811.0) - subdwarfs white dwarfs

\section{Introduction}

Hot subdwarfs (sdOs/sdBs) are low-mass He-stars with very thin hydrogen envelopes. They have effective temperatures similar to $\mathrm{O}$ and $\mathrm{B}$ stars but are several orders of magnitude less luminous due to their small size (Heber 1986, 2009, 2016). Maxted et al. (2001) and Napiwotzki et al. (2004) showed that $>50 \%$ of $\mathrm{sdB} / \mathrm{sdO}$ stars are in compact binaries with orbital periods $P_{\text {orb }}<10$ days. Formation and orbital shrinkage through a common envelope phase are the only way to form such compact binaries (Han et al. 2002, 2003; Nelemans 2010).

It has been shown that hot subdwarfs in compact $\mathrm{sdB} / \mathrm{sdO}+$ white dwarf (WD) binaries with $P_{\text {orb }} \lesssim 2 \mathrm{hr}$ on the exit of the common envelope phase still burn helium when the $\mathrm{sdB} / \mathrm{sdO}$ fills its Roche Lobe (RL). Due to the emission of gravitational waves, the binary is predicted to shrink until the hot subdwarf star fills its RL at an orbital period of 16-50 minutes and starts mass-transfer. He-rich material is then transferred to the WD companion with typical mass-transfer rates of $\dot{M} \approx 10^{-8} M_{\odot} \mathrm{yr}^{-1}$ (e.g., Savonije et al. 1986; Tutukov \& Fedorova 1989; Tutukov \& Yungelson
1990; Iben \& Tutukov 1991; Yungelson 2008; Piersanti et al. 2014; Brooks et al. 2015).

Most of the known compact $\mathrm{sdB} / \mathrm{sdO}$ binaries reside in systems with orbital periods $\geqslant 0.1$ days where the $\mathrm{sdB} / \mathrm{sdO}$ will have turned into a carbon/oxygen WD when both components come into contact (Kupfer et al. 2015). So far, only two hot subdwarf binaries with a white dwarf companion and an orbital period $P_{\text {orb }}<90$ minutes are known (Vennes et al. 2012; Geier et al. 2013; Kupfer et al. 2017).

Just recently, Kupfer et al. (2017) discovered the ultracompact sdB+WD binary, PTF1 J082340.04+081936.5, with $P_{\text {orb }}=87$ minutes. The object is close to the limit to start future accretion, while the $\mathrm{sdB}$ is still burning helium. If the sdB still burns helium when the system comes into contact, helium-rich material will be accreted onto the WD companion and the most likely outcome is a helium-accreting AMCVn-type system, because the companion is by then a low-mass white dwarf $\left(M_{\mathrm{WD}}=0.46_{-0.09}^{+0.12} M_{\odot}\right)$.

The most compact sdB binary with a WD companion is $\mathrm{CD}-30^{\circ} 11223\left(P_{\text {orb }}=70.5\right.$ minutes, Vennes et al. 2012; 
Geier et al. 2013). The sdB will fill its $\mathrm{RL}$ in $\approx 40$ million years, well within the He-burning lifetime of the sdB star. The WD companion is massive $\left(M_{\mathrm{WD}} \approx 0.75 M_{\odot}\right)$ and after accreting $0.1 M_{\odot}$, He-burning is predicted to be ignited unstably in the accreted helium layer on the surface of the white dwarf (Brooks et al. 2015; Bauer et al. 2017). This could trigger the ignition of carbon in the core, which might disrupt the WD even when the mass is significantly below the Chandrasekhar mass, a so-called double-detonation supernova type Ia (e.g., Livne 1990; Livne \& Arnett 1995; Fink et al. 2010; Woosley \& Kasen 2011; Wang et al. 2013; Shen \& Bildsten 2014). The hot subdwarf will become unbound and ejected with the orbital velocity, which can be up to $\approx 1000 \mathrm{~km} \mathrm{~s}^{-1}$. The hypervelocity sdO star US708 (Hirsch et al. 2005) has been proposed as a candidate for such a donor remnant (Geier et al. 2013, 2015).

Bildsten et al. (2007) showed that unstable He shell-burning can detonate the He shell without disrupting the WD, which can be observed as a faint and fast Ia supernova. The detonation will increase the orbit and the binary system will lose contact. Gravitational wave radiation will decrease the orbit again and bring both objects back into contact, which can trigger several subsequent weaker flashes (Brooks et al. 2015).

The OmegaWhite survey is a high-cadence synoptic survey of the southern Galactic Plane and Galactic Bulge, the main aim of which is to identify ultracompact binaries (Macfarlane et al. 2015; Toma et al. 2016; Macfarlane et al. 2017a, 2017b). OmegaWhite observations are obtained using the VST telescope at the European Southern Observatory's (ESO) Paranal site in Chile. Each field is imaged an average of 38 times over a $2 \mathrm{hr}$ period, with $39 \mathrm{~s}$ integrations. Light curves are extracted from image differencing and a Lomb-Scargle and AoV analysis is performed on all extracted light curves to identify short-period variables. OW J074106.0-294811.0 (OW J0741 hereafter) was discovered as a faint $(g=20.0)$ blue $(u-g=-1.23 \mathrm{mag}, g-r=0.18 \mathrm{mag})$ source, photometrically variable on a period of 22.6 minutes in the OmegaWhite survey (Macfarlane et al. 2015). It was noted in Toma et al. (2016) that further observations of OW J0741 showed that it was a binary with a 44-minute orbital period. Here, we report the discovery of OW J0741 as an ultracompact sdOB + WD binary with an orbital period of $P_{\text {orb }}=44.66$ minutes, making it the most compact hot subdwarf binary known today.

The paper is structured as follows. Section 2 describes the observations used in the analysis. The orbital and binary parameter, as well as the atmospheric parameters, are described in Sections 3 and 4. The light curve analysis and the system parameters are presented in Sections 5 and 6 . Section 7 discusses the structure and evolutionary history of the system using MESA. A summary and conclusions are given in Section 8.

\section{Observations}

\subsection{Optical Photometry}

Optical photometric data were obtained using the South African Astronomical Observatory (SAAO) $1.9 \mathrm{~m}$ Telescope with the Sutherland High Speed Optical Camera (SHOC), Keck/LRIS, Gemini South/GMOS as well as the $3.5 \mathrm{~m} \mathrm{New}$ Technology Telescope (NTT) with ULTRACAM and the Southern African Large Telescope (SALT) with SALTICAM.
Photometry from the SAAO was obtained using the $1.9 \mathrm{~m}$ telescope and SHOC (Coppejans et al. 2013), which uses an Andor E2V CCD and works in frame-transfer mode (with $1024 \times 1024$ active pixels) so that the readout time is effectively zero. It has been designed to run at up to 20 frames $\mathrm{s}^{-1}$ with high-accuracy timing for each frame, but because this object is so faint, typical exposure times were 30-40 s (see Table 1). No filter was used in order to maximize the count rate, and the SHOC data were reduced using an in-house SAAO aperture photometry pipeline based on IRAF routines. On the $1.9 \mathrm{~m}$ telescope, SHOC has a field-of-view of $2 ! 8 \times 2 ! 8$, so several brighter stars were available for differential photometry. We used three photometrically stable field stars for photometric calibration. The observations were conducted under partly cloudy conditions, with an average seeing of 1 !! 2 .

Observations of OW J0741 were made on 2016 December 7 using the high-speed photometer SALTICAM (see O'Donoghue et al. 2006) mounted on SALT at the Sutherland Observatory in South Africa. Observations were made with no filter and an exposure time of $2 \mathrm{~s}$ and the data set consists of 1470 images. The SALTICAM frame-transfer mode allows for exposures with $200 \mathrm{~ms}$ readout time.

Photometric follow-up observations were obtained with Keck and Gemini South using LRIS (Oke et al. 1995) and GMOS (Hook et al. 2004) in imaging mode, respectively. Both setups used the $g$ ' filter and a $20 \mathrm{~s}$ exposure time. For GMOS we used a $2 \times 2$ binning and a $1 \times 1$ arcmin window to reduce the readout time to $\approx 10 \mathrm{~s}$. Gemini observed OW J0741 at an airmass $\approx 1.05$ with an average seeing of 0 ." 7 . The Keck light curve was obtained at an airmass of $\approx 1.5$ and an average seeing of 1 !" 1 . The readout time of the CCD was $42 \mathrm{~s}$ for each exposure.

High-cadence observations were obtained using ULTRACAM (Dhillon et al. 2007) on the ESO $3.58 \mathrm{~m}$ NTT on 2016 December 6. ULTRACAM is a high-speed photometer that observes in three color bands simultaneously and uses frame-transfer CCDs to reduce dead time between exposures to almost zero. Sloan filters $u$ ', $g$ ', and $r^{\prime}$ were used. The exposure time was in the beginning $10 \mathrm{~s}$ and later $20 \mathrm{~s}$ in $g^{\prime}$ and $r^{\prime}$, and 20 and $40 \mathrm{~s}$ in $u^{\prime}$ to compensate for the lower throughput of that band. These observations covered two full orbits of the system under clear conditions, with an average seeing of $1^{\prime \prime}$. Each image was biasand dark-subtracted, and divided through by a twilight flat-field.

The photometric data from Keck, Gemini, and ULTRACAM were reduced using the ULTRACAM pipeline. The count rate of OW J0741 was extracted using differential aperture photometry, using the same comparison star for Keck, Gemini, and ULTRACAM to remove atmospheric transparency effects.

An aperture of $1.7 \times$ the FWHM of the star was used to extract the photometry in the Keck and Gemini data. For the ULTRACAM data, the aperture width was reduced to $1.5 \times$ the FWHM of the star, in order to avoid contamination from nearby stars due to the lower resolving power of the NTT.

\subsection{Spectroscopy}

\subsubsection{ESO VLT}

We obtained longslit spectroscopy of OW J0741 using the FORS2 instrument (Appenzeller et al. 1998) at the ESO VLT UT1. We used grism 600B with a $1^{\prime \prime}$ slit width, for a spectral 
Table 1

Summary of the Observations of OW J0741

\begin{tabular}{|c|c|c|c|c|c|}
\hline Date & UT & Tele./Inst. & $N_{\exp }$ & Exp. Time (s) & Coverage $(\AA) /$ Filter \\
\hline \multicolumn{6}{|l|}{ Photometry } \\
\hline 2016 Apr 14 & $17: 47-20: 29$ & SAAO/SHOC & 325 & 30 & clear \\
\hline 2016 Apr 15 & $17: 13-19: 22$ & SAAO/SHOC & 257 & 30 & clear \\
\hline 2016 Apr 18 & $17: 04-19: 29$ & SAAO/SHOC & 216 & 40 & clear \\
\hline 2016 Apr 19 & $18: 28-19: 58$ & SAAO/SHOC & 136 & 40 & clear \\
\hline 2016 Nov 03 & $14: 47-15: 39$ & Keck/LRIS & 56 & 20 & $g^{\prime}$ \\
\hline 2016 Nov 28 & $05: 30-06: 32$ & Gemini/GMOS & 95 & 23 & $g^{\prime}$ \\
\hline 2016 Dec 05 & $05: 37-06: 39$ & Gemini/GMOS & 95 & 23 & $g^{\prime}$ \\
\hline 2016 Dec 06 & $05: 32-06: 35$ & Gemini/GMOS & 95 & 23 & $g^{\prime}$ \\
\hline 2016 Dec 07 & 05:32-07:00 & NTT/ULTRACAM & 207 & $20 / 40$ & $u^{\prime}$ \\
\hline 2016 Dec 07 & $05: 32-07: 00$ & NTT/ULTRACAM & 412 & $10 / 20$ & $g^{\prime} r^{\prime}$ \\
\hline 2016 Dec 07 & $21: 57-22: 45$ & SALT/Salticam & 1470 & 2 & clear \\
\hline \multicolumn{6}{|l|}{ Spectroscopy } \\
\hline 2016 Sep 20 & $08: 37-09: 27$ & VLT/FORS2 & 9 & 300 & $3500-6300$ \\
\hline 2016 Nov 03 & 06:38-08:07 & Gemini/GMOS & 20 & 240 & $3500-6700$ \\
\hline 2016 Nov 03 & $13: 22-14: 08$ & Keck/LRIS & 10 & 240 & $3400-5600$ \\
\hline 2016 Nov 04 & $05: 39-06: 23$ & Gemini/GMOS & 10 & 240 & $3500-6700$ \\
\hline 2016 Nov 28 & $12: 00-13: 38$ & Keck/LRIS & 20 & 240 & $3400-5600$ \\
\hline 2016 Dec 29 & $10: 00-10: 41$ & Keck/LRIS & 9 & 240 & $3400-5600$ \\
\hline 2017 Jan 26 & 09:15-10:01 & Keck/LRIS & 10 & 240 & $3400-5600$ \\
\hline \multicolumn{6}{|l|}{ Swift } \\
\hline 2016 Oct 24 & 04:06-06:02 & XRT & 1 & 2944 & $0.2-10 \mathrm{keV}$ \\
\hline 2016 Oct 24 & 04:06-06:02 & UVOT & 1 & 2922 & 1600-2260 (UVW2) \\
\hline
\end{tabular}

resolution of $\sim 1000$ (measured on the arc lines), and we obtained 9 spectra on the night 2016 September 20 between 08:37 and 09:27 UT, each of them with an exposure time of $300 \mathrm{~s}$. The original pixel size was $1.38 \AA$, but to increase the signal-to-noise $(\mathrm{S} / \mathrm{N})$ we smoothed the spectra with a running average 6 pixels wide, effectively reducing the spectral resolution to $\sim 600$. The ambient condition report shows that the night was photometric and that during the observations seeing varied from $1^{\prime \prime}$ to 1 !' 25 . The peak $\mathrm{S} / \mathrm{N}$ of individual spectra was $\sim 8$ per $\AA$.

Data reduction was performed using standard IRAF routines. We used spectrophotometric standard stars obtained during nights close to that of the observations of the main target. White dwarf LDS 749B (=LAWD 87) was observed on 2016 September 18 at UT 03:26, and white dwarf EGGR 21 (=CPD-69 177) was observed twice consecutively on 2016 September 22 at UT 08:23 and 08:26 within the context of the standard FORS calibration plan. Calibrated fluxes were taken from Oke (1990) for LDS 749B and from Hamuy et al. (1992, 1994) for EGGR 21. The observations of the standard stars were obtained in MOS mode with a slit of $5^{\prime \prime}$ width, located in a slightly offset position with respect to the $1^{\prime \prime}$ longslit used for the main target, with the consequence that the spectral coverage of the standard star does not perfectly overlap that of the target, and the transmission function at wavelengths longer than $6000 \AA$ had to be extrapolated. Fluxes were corrected for atmospheric extinction using Paranal extinction coefficients from Patat et al. (2011). The correction of the instrumental sensitivity obtained with the two different data sets of standard stars led to consistent results, and in the end we adopted only star EGGR 21 as a calibrator. The use of a $1^{\prime \prime}$ slit width while seeing conditions were about 1 !' 25 led to wavelengthdependent slit losses up to $30 \%$.

\subsubsection{Gemini South}

Optical spectra were obtained using Gemini South with the GMOS spectrograph over 2 nights using a low-resolution mode $(R \approx 1500)$. Twenty consecutive spectra were obtained on 2016 November 3 and 10 consecutive spectra were obtained on 2016 November 4. An average bias frame was made out of five individual bias frames and a normalized flat-field frame was constructed out of six individual lamp flat-fields. $\mathrm{CuAr}$ arc exposures were taken before and after 10 consecutive spectra to correct for instrumental flexure. Each exposure was wavelength-calibrated by interpolating between the two closest exposures. All spectra were reduced using the IRAF package for GMOS.

\subsubsection{Keck}

Over four nights, we obtained a total of 49 spectra using the Keck I telescope and the LRIS spectrograph (Oke et al. 1995) in a low-resolution mode $(R \approx 750)$. Ten bias frames were obtained to construct an average bias frame and ten individual lamp flat-fields were obtained to construct a normalized flatfield. $\mathrm{HgNeArCaSn}$ arc exposures were taken before and after each observing sequence. Each exposure was wavelengthcalibrated by interpolating between the two closest exposures and skylines were used to correct for instrumental flexure. All spectra were reduced using a custom IDL-based package.

\subsection{X-Ray and UV Observations}

We obtained Director's Discretionary Time on the Swift satellite on 2016 October 24, giving exposure times of $2944 \mathrm{~s}$ and $2922 \mathrm{~s}$ on the X-ray Telescope (XRT) and UVOT (UVW2 filter), respectively. The XRT is sensitive over the range $0.2-10 \mathrm{keV}$ and we examined data taken in "photon counting" mode and used the products derived from the standard XRT pipeline. 
An overview of all our observations is given in Table 1.

\section{Orbital and Binary Parameters}

The photometric data that led to the discovery of OW J0741 were a $2.5 \mathrm{hr}$ sequence of $44 \times 39 \mathrm{~s}$ exposures in the $g$ '-band as part of the OmegaWhite survey (Macfarlane et al. 2015). This light curve showed a strong peak in its power spectrum at 22.6 minutes and a corresponding amplitude of 0.22 mag (Toma et al. 2016). The SAAO $1.9 \mathrm{~m}$ telescope and SHOC in 2016 April (see Table 1) show unequal minima on top of the predominantly sinusoidal variability. The dominant modulation in the light curve is caused by ellipsoidal deformation of the sdOB and the unequal minima are caused due to gravitydarkening of the deformed sdOB. The unequal depth of the minima was confirmed when we obtained $8 \mathrm{~m}$ class photometric data from Keck and Gemini.

To determine an ephemeris we initially determined the time of the deepest minima in the Keck and Gemini light curves by eye. This allowed us to assign an unambiguous cycle number to each minimum and remove any secondary minima. We then determined a linear fit to these times assuming a constant period. This allowed us to determine the period of OW J0741 to better than $0.1 \mathrm{~s}$. We were then able to assign a cycle number to the minima in the light curve of the SAAO data obtained in 2016 April and the ULTRACAM data taken in 2016 December. A linear fit to these minima gives an ephemeris of

$$
\begin{aligned}
T_{o}(\mathrm{HMJD})= & 57695.611284 \pm 0.000166 \\
& +0.031015829 \pm\left(7.1 \times 10^{-8}\right) E .
\end{aligned}
$$

We are not able to use the OmegaWhite data taken in 2012 March to refine this further because there is an ambiguity regarding which of the observed minima are the deepest minima.

The individual spectra that we obtained for OW J0741 all have a relatively low $\mathrm{S} / \mathrm{N}(\lesssim 10)$ and hence are not well suited for searching for a radial velocity (RV) period. We therefore folded the spectra of OW J0741 on the ephemeris shown in Equation (1) into 14 phase-bins and co-added individual spectra observed at the same binary phase. This increased the $\mathrm{S} / \mathrm{N}$ per phase-bin to $\approx 25$.

We used the FITSB2 routine (Napiwotzki et al. 2004) to measure the velocities. FITSB2 fits Gaussians, Lorentzians, and polynomials to the hydrogen and helium lines to fit the continuum, line, and line core of the individual lines. Using a $\chi^{2}$-minimization we fitted the wavelength shifts compared to the rest wavelengths of all suitable spectral lines (Geier et al. 2011). Assuming circular orbits, a sine curve was fitted to the folded RV data points. We find a semi-amplitude $K=422.5 \pm$ $21.5 \mathrm{~km} \mathrm{~s}^{-1}$ and a systemic velocity of $\gamma=-14.0 \pm$ $11.5 \mathrm{~km} \mathrm{~s}^{-1}$ (Figure 1), making it the most compact hot subdwarf binary with the largest RV amplitude.

\section{Atmospheric Parameters of the Hot Subdwarf Star}

The atmospheric parameters of effective temperature $T_{\text {eff }}$, surface gravity, $\log g$, and helium abundance, $\log y$, where $y=n(\mathrm{He}) / n(\mathrm{H})$, were determined for the sdOB by fitting the rest-wavelength-corrected average spectra with metal-free NLTE model spectra (Stroeer et al. 2007). To obtain the average spectrum we shifted the 14 phase-binned spectra that were used for the RV measurement to the rest-wavelength and calculated the combined average spectrum. The spectrum

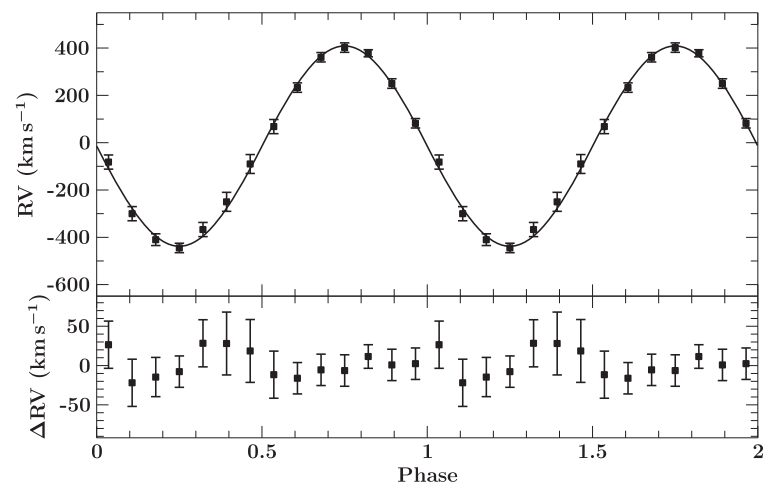

Figure 1. Radial velocity plotted against orbital phase. The RV data were phase-folded with the orbital period and are plotted twice for better visualization. The residuals are plotted below.

shows Balmer lines as well as neutral (He I) and ionized (He II) helium lines (see Figure 2). The ionization equilibrium between the $\mathrm{He} \mathrm{I}$ and $\mathrm{He}$ II is most sensitive to the effective temperature of the sdO, whereas the broad He II and the hydrogen lines in the blue are most sensitive to $\log g$. We find $T_{\text {eff }}=$ $39400 \pm 500 \mathrm{~K}, \log g=5.74 \pm 0.09$, and $\log y=-0.14 \pm$ 0.07 (Figure 3). The occurrence of both $\mathrm{He}$ I and He II as well as the increased helium abundance $\log y>-1$ classifies the hot subdwarf as intermediate He-sdOB (see Heber 2016). The errors were derived using a $\chi^{2}$-minimization.

\section{Light Curve Analysis}

The LCURVE code was used to perform the light curve analysis (Copperwheat et al. 2010). LCURVE uses grids of points to model the two stars. The shape of the stars in the binary is set by a Roche potential. We assume that the orbit is circular and that the rotation periods of the stars are synchronized to the orbital period. The flux that each point on the grid emits is calculated by assuming a blackbody of a certain temperature at the bandpass wavelength, corrected for limb-darkening, gravity-darkening, Doppler-beaming, and the reflection effect.

Some additional information was used as input. The orbital period was fixed to 44.66279 minutes as derived in Section 3. From spectroscopy the $T_{\text {eff }}, \log g$, and semi-amplitude $K$ were fixed to the values derived in Sections 3 and 4. Additionally, as a lower limit to the radius (mass) of the white dwarf companion, we use the zero-temperature mass-radius relation by Eggleton (quoted from Verbunt \& Rappaport 1988). The passband specific gravity-darkening was calculated as described in Bloemen et al. (2011). We use $\beta=0.33 \pm 0.01$ in $g^{\prime}$ and $\beta=0.34 \pm 0.01$ in $r$ '. The limb-darkening coefficients were calculated with the Claret limb-darkening prescription (Claret 2004). We used $\mathrm{a}_{1}=0.613, \mathrm{a}_{2}=-0.645$, $\mathrm{a}_{3}=0.621$, and $\mathrm{a}_{4}=-0.239$ for $g$ ' and $\mathrm{a}_{1}=0.422$, $\mathrm{a}_{2}=-0.444, \mathrm{a}_{3}=0.475$, and $\mathrm{a}_{4}=-0.194$ for $r$ '. This leaves as free parameters in the model the mass ratio $q=\frac{M_{\mathrm{sdOB}}}{M_{\mathrm{WD}}}$, the inclination $i$, secondary temperature $T_{\mathrm{WD}}$, the scaled radii of both components $r_{\mathrm{sdOB} / \mathrm{WD}}$, the velocity scale $\left(\left[K+K_{\mathrm{WD}}\right] / \sin i\right)$ and the beaming parameter $B \quad\left(F_{\lambda}=\right.$ $F_{0, \lambda}\left[1-B \frac{v_{r}}{c}\right]$, see Bloemen et al. 2011). In addition, to account for any residual airmass effect we add a third-order polynomial.

To determine the uncertainties in the parameters we combine LCURVE with emcee (Foreman-Mackey et al. 2013), an 

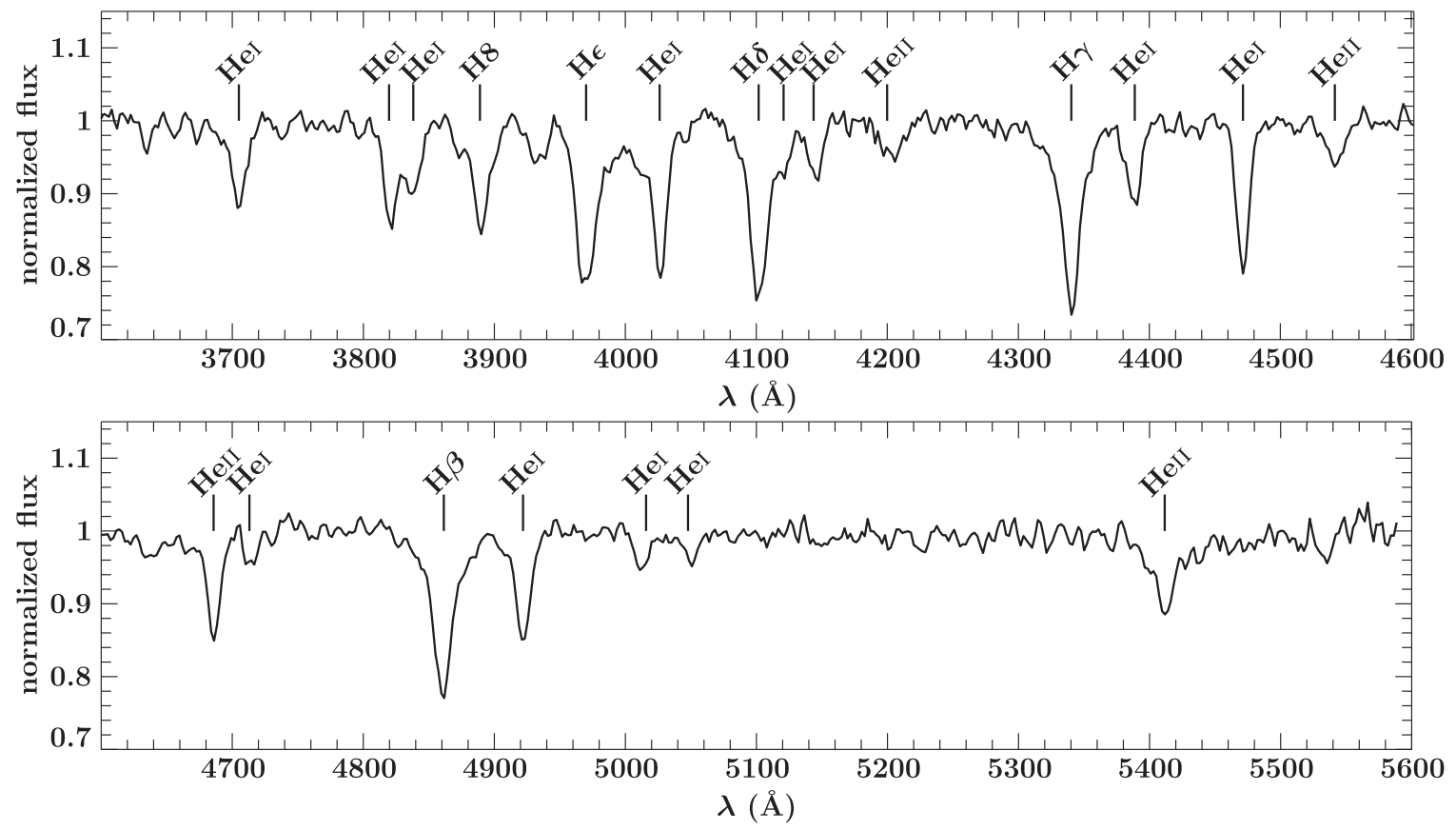

Figure 2. Average spectrum of OW J0741. All prominent lines are marked.

implementation of an MCMC sampler that uses a number of parallel chains to explore the solution space. We use 256 chains and let them run until the chains stabilized to a solution, which took approximately 1000 generations.

For the Keck and ULTRACAM $g$ '-band and $r$ '-band light curves we find two distinct solutions: one that finds the system eclipsing and the other that finds the system non-eclipsing (Figure 4). The Gemini light curves have a higher precision and we find exclusively non-eclipsing solutions (Figure 5). This becomes particularly obvious in the significant larger error bars for inclination $(i)$, mass ratio $(q)$, and sdOB mass $\left(M_{\mathrm{sdOB}}\right)$ for the individual fits to the Keck and ULTRACM light curves (Table 2). To obtain the final solution we fit all six light curves simultaneously. From the simultaneous fit we only find noneclipsing solutions (Figure 6).

\section{System Parameters}

From the strong light curve variability caused by ellipsoidal modulation in combination with the RV amplitude and the spectroscopic solution, we can solve the system and derive system parameters. Solutions were calculated for each individual light curve, as well as from a simultaneous fit to all six light curves. The results from the latter are taken as the final solution (Table 2).

We find that the system consists of a low-mass sdOB with a high-mass WD companion. A mass ratio $q=M_{\mathrm{sdOB}} / M_{\mathrm{WD}}=$ $0.32 \pm 0.10$, a mass for the $\mathrm{sdOB} M_{\mathrm{sdOB}}=0.23 \pm 0.12$ $M_{\odot}$, and a WD companion mass $M_{\mathrm{WD}}=0.72 \pm 0.17 M_{\odot}$ were derived. The inclination is found to be $i=$ $57^{\circ} .4 \pm 4.7$ (Table 3 ).

We calculate the distance to OW J0741 using the visual $V$ magnitude $\left(m_{\mathrm{V}}\right)$, the sdOB mass $\left(M_{\mathrm{sdOB}}\right), T_{\text {eff }}$ and $\log g$ as described in Ramspeck et al. (2001). The visual $V$ magnitude $m_{\mathrm{V}}=20.06 \mathrm{mag}$ was calculated following the conversion from SDSS colors as described in Jester et al. (2005). Because OW J0741 is located toward the Galactic Bulge, significant reddening can occur. Green et al. (2015) calculated
3D extinction maps from PanSTARRS data ${ }^{16}$ and find toward the direction of OW J0741 an extinction of $E(B-V)=$ $0.34_{-0.034}^{+0.032}$ at distances above $6 \mathrm{kpc}$, resulting in a total extinction in the $V$-band of $A_{V}=1.05 \mathrm{mag}$ using $R_{V}=3.1$. With the corrected magnitude we find a distance of $d=6.6 \pm 2.1 \mathrm{kpc}$, which for a Galactic latitude of -3.5 gives a height below the Galactic plane of $\sim 400 \mathrm{pc}$.

For the X-ray observations no source was detected with a $3 \sigma$ upper limit of $0.0036 \mathrm{ct} \mathrm{s}^{-1}$. Assuming a $1 \mathrm{keV}$ thermal bremsstrahlung and a Galactic absorption of $4.4 \times 10^{21} \mathrm{~cm}^{-2}$ (the average to the edge of the Galaxy) we find an upper limit of $2.6 \times 10^{-13} \mathrm{erg} \mathrm{cm}^{-2} \mathrm{~s}^{-1}$ to the unabsorbed flux (this limit is not strongly dependent on the assumed model). Given the large distance to OW J0741, the upper limit for the X-ray luminosity $\left(<2 \times 10^{33} \mathrm{erg} \mathrm{s}^{-1}\right)$ is not very constraining, since the upper end of X-ray luminosity of hot subdwarfs is $\sim 10^{31} \mathrm{erg} \mathrm{s}^{-1}$ (Mereghetti \& La Palombara 2016). In contrast, OWJ0741 was detected in the UVW2 filter (peak effective area $1928 \AA$ ) with a corrected count rate of $0.34 \pm 0.01 \mathrm{ct} \mathrm{s}^{-1}$, implying a flux $2.04 \pm 0.08 \times 10^{-16} \mathrm{erg} \mathrm{cm}^{-2} \mathrm{~s}^{-1} \AA^{-1}$.

\section{Discussion}

\subsection{Origin of the sdOB Star}

Using the constraints from the observations outlined above, we use the stellar evolution code MESA (Paxton et al. 2011, $2013,2015)$ to put constraints on the nature of the sdOB star. We then take the most likely model and evolve it forward in time to generate predictions about the future of this system.

\subsubsection{He-star Model}

We first test a model with the canonical sdOB mass of $0.462 M_{\odot}$ with a hydrogen envelope mass of $2.5 \times 10^{-4} M_{\odot}$. We find that after core He-burning and during shell $\mathrm{He}$ burning, this model spends $\approx 4 \mathrm{Myr}$ in crossing the observation

\footnotetext{
${ }^{16}$ http://argonaut.skymaps.info/
} 

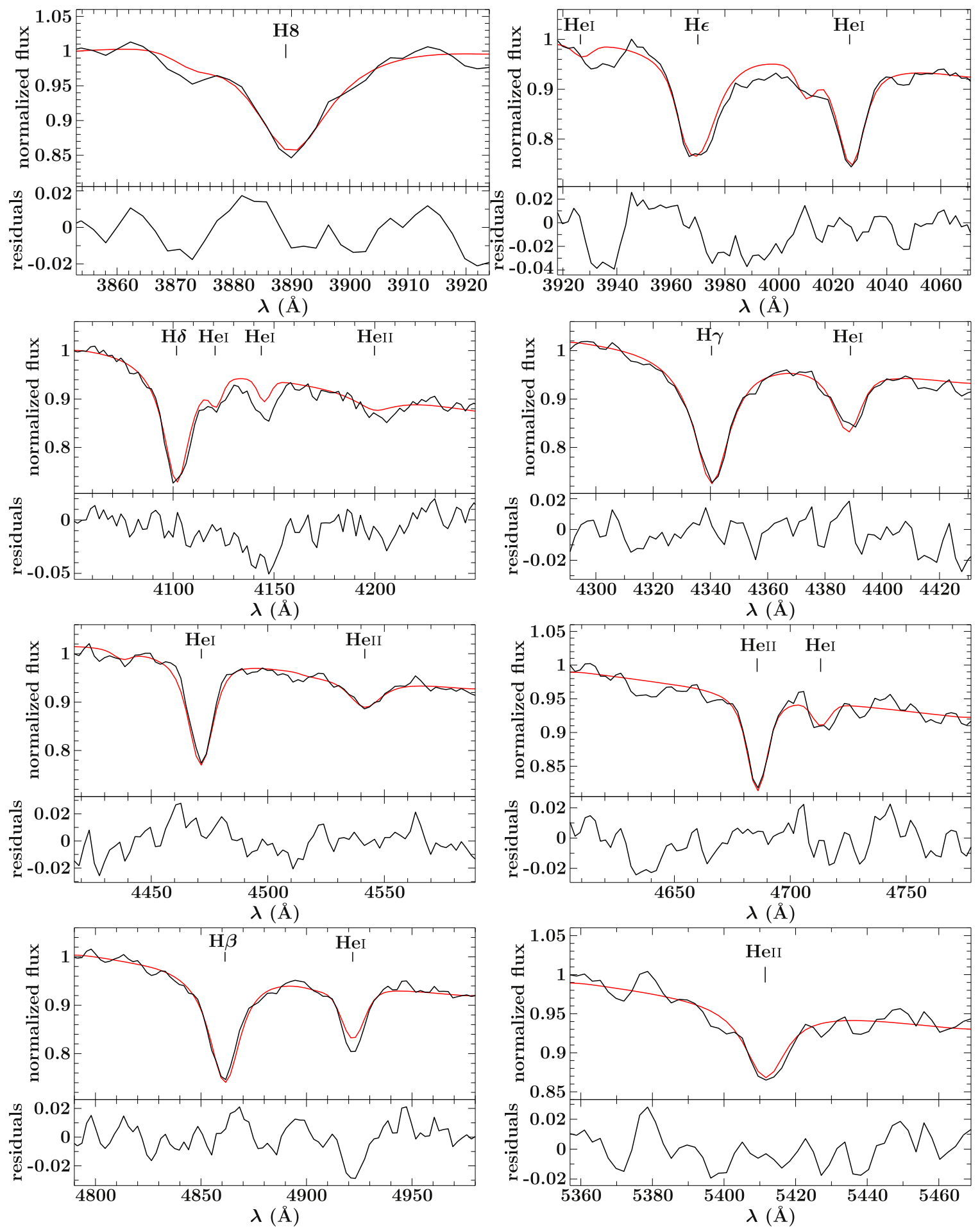

Figure 3. Fit of synthetic NLTE models to the hydrogen Balmer line, as well as neutral and ionized helium lines of the co-added spectrum.

box (see dot-dashed purple line in Figure 7). There are a few problems with the system being in this state. First, given the $\log g$, orbital period, and mass ratio, this model fills its RL at orbital periods $>50$ minutes, meaning that the higher mass (compared to the derived mass from observations) implies a radius larger than the current RL. ${ }^{17}$ Second, the

\footnotetext{
17 Given the length of the He core-burning phase, this system would have a wider orbit during He core-burning and avoid Roche Lobe overflow (RLOF) at that phase of evolution.
}

derived mass ratio from observations implies that if the He-star had a mass of $0.46 M_{\odot}$, the compact companion must be super-Chandrasekhar, as well as that the derived mass from the observations is inconsistent with the canonical sdOB mass.

We also tested He-star models between $0.325 M_{\odot}$ and $0.462 M_{\odot}$ that experienced He core-burning, and found that during the He core-burning stage, $T_{\text {eff }}$ was too low and $\log g$ was too high to match the measurements. If the models were able to produce a $\mathrm{He}$-star massive enough to experience $\mathrm{He}$ 

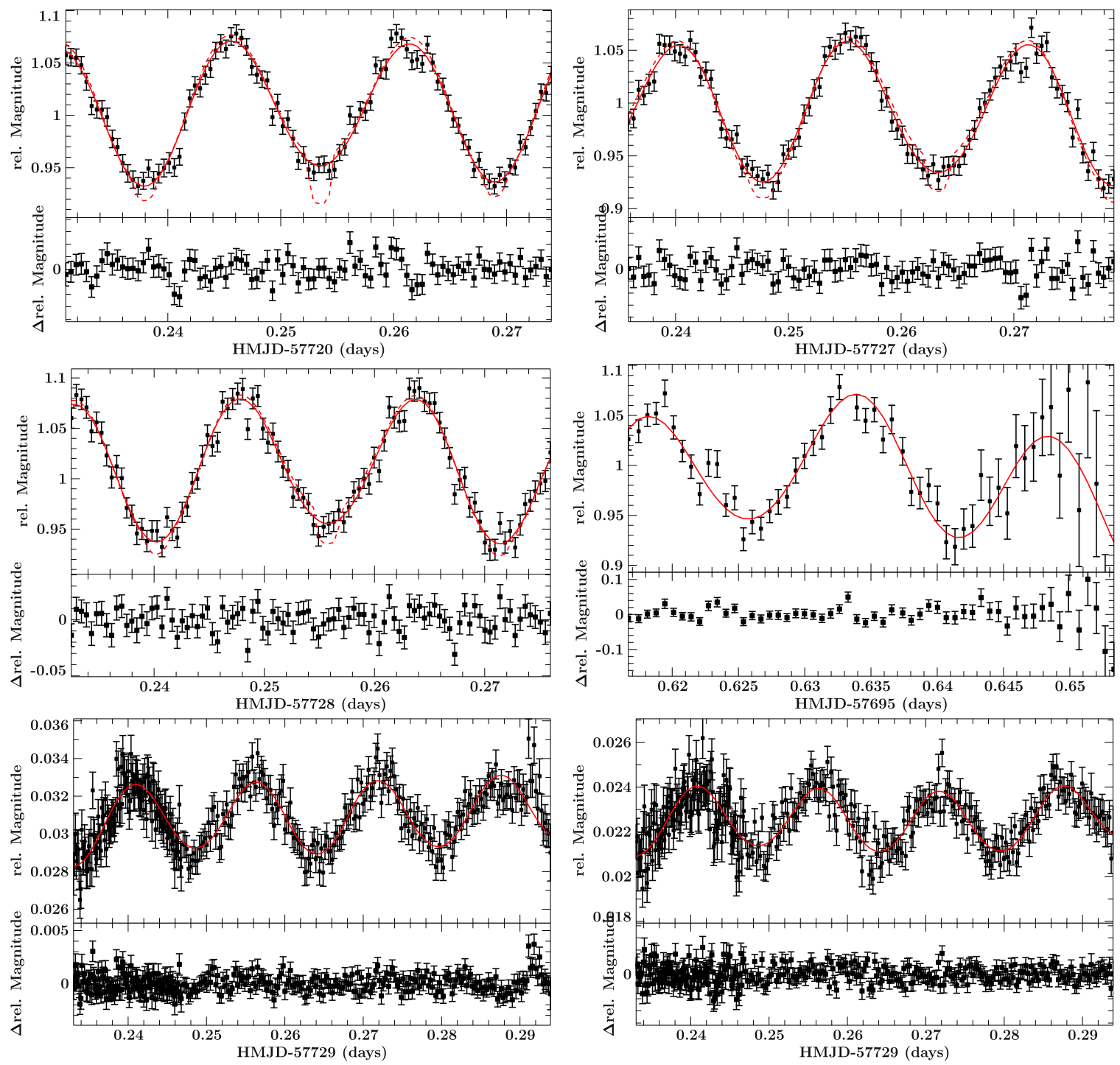

Figure 4. Light curves shown together with the LCURVE fits. For the Gemini light curves, the non-eclipsing (solid) and the eclipsing solution (dotted) are shown. The non-eclipsing solution is shown for the Keck and ULTRACAM light curves. The residuals are plotted below. Upper left: Gemini (2016 November 28). Upper right: Gemini (2016 December 05). Middle left: Gemini (2016 December 06). Middle right: Keck (2016 November 03). Lower left: ULTRACAM $g$ ' (2016 December 07). Lower right: ULTRACAM $r^{\prime}$ (2016 December 07).

shell-burning, this stage reached higher $T_{\text {eff }}$ values, but with similar $\log g$ values to the He core-burning stage. Therefore, only He shell-burning models near $0.462 M_{\odot}$ were able to match the $T_{\text {eff }}$ and $\log g$ measurements.

\subsubsection{He White Dwarf Model}

In a second scenario, we consider the sdOB star as a helium white dwarf (He WD) that did not start helium-burning. If we assume that the star is a He WD that is just coming out of a CE event, then the $T_{\text {eff }}$ and $\log g$ observation box gives a solution of a unique mass (Althaus et al. 2013). Figure 7 shows that this mass must be close to $0.320 M_{\odot}$, shown by the middle solid curve. This model spends $\approx 220,000$ years in the observation box and has a post-CE age of $\approx 1.1 \mathrm{Myr}$.

$\mathrm{He}$ WDs of this mass range experience diffusion-induced $\mathrm{H}$ novae (Althaus et al. 2001), and the tracks of these novae pass through the same $\log g$ values, but at higher $T_{\text {eff }}$ for a given stellar mass. Therefore, we can construct a lower-mass He WD model $\left(0.242 M_{\odot}\right)$ that has low $T_{\text {eff }}$ just out of the CE but passes through the observation box after the first diffusioninduced $\mathrm{H}$-nova. This is shown by the orange dashed curve in Figure 7. This model spends $\approx 66,000$ years in the observation box and has a post-CE age of $\approx 11 \mathrm{Myr}$. Since the $0.320 M_{\odot} \mathrm{He}$ WD model spends more than a factor of 3 more time in the observation box, we conclude that the $0.320 M_{\odot}$ model is the more likely fit to our observations, even though the lower-mass He WD model fits the derived mass better.

\subsection{Future Predictions}

To predict the future of OW J0741, we use our best-fit model for the sdOB, a $0.320 M_{\odot} \mathrm{He} \mathrm{WD}$, and given the derived mass ratio, a $0.85 M_{\odot}$ WD companion. As mentioned above, WDs in the mass range considered for the sdOB experience diffusioninduced hydrogen novae. This model experiences its first H-nova 4.1 Myr after passing through the observation box and expands to fill its RL at an orbital period of 40.6 minutes. Depending on the amount of mass lost through RLOF during 

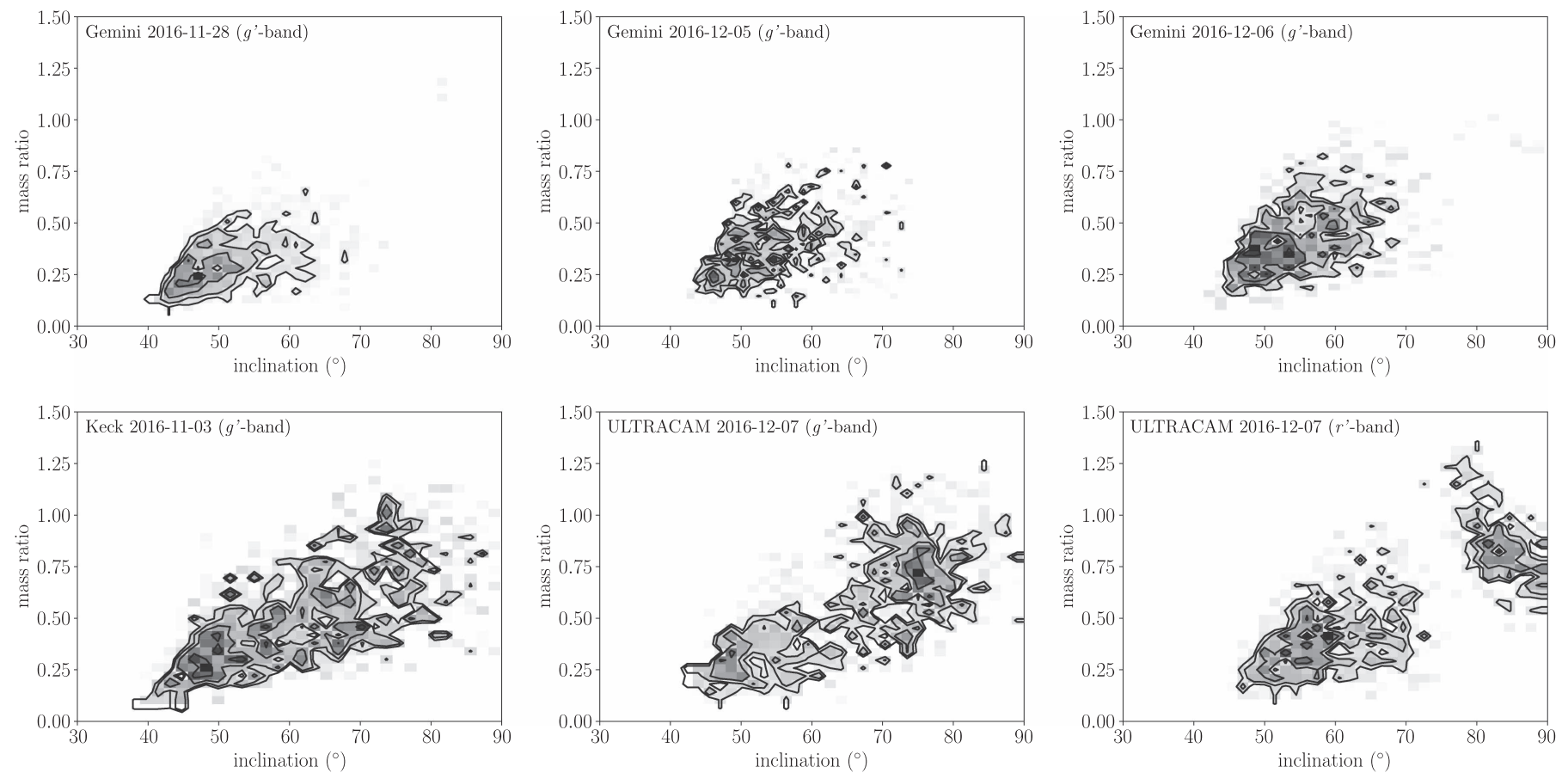

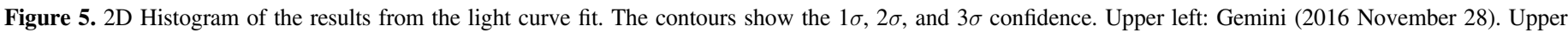

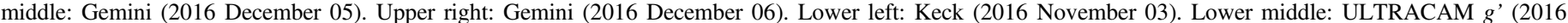
December 07). Lower right: ULTRACAM r' (2016 December 07).

this hydrogen nova, the model may experience a second hydrogen nova.

The subsequent evolution is governed by gravitational wave radiation, which shrinks the orbit. Because the WD contraction occurs faster than the orbital decay, the He WD begins RLOF after 17.6 Myr, at an orbital period of 5 minutes. For the given mass ratio, OW J0741 is definitely stable according to the dynamical instability (Nelemans et al. 2001), but definitely unstable according to the mass ratio criterion for direct impact accretion. Therefore, whether or not this system will be stable to mass-transfer depends on the spin-orbit synchronization timescale, $\tau_{s}$ (Marsh et al. 2004), of the accreting WD. The physics that determine the value of $\tau_{s}$ is uncertain and previous estimates for systems of this type differ by more than 10 orders of magnitude (Campbell 1983, 1984; Fuller \& Lai 2014).

According to Figures 1 and 5 from Marsh et al. (2004), the stability of this system requires a synchronization timescale of $\tau_{s} \lesssim 10$ year. If the synchronization timescale is longer than this, then the WD accretor will extract enough of the orbital angular momentum into its spin angular momentum to cause a merger, leading to an $\mathrm{R} \mathrm{CrB}$ configuration. If instead the synchronization timescale is short enough, the spin of the accreting WD can couple to the orbit and feedback enough angular momentum to avoid a merger, leading to a stable mass transferring system. In this case, the He WD itself will begin transferring degenerate helium at an orbital period of 3 minutes, leading to mass-transfer rates of $\approx 3 \times 10^{-6} M_{\odot} \mathrm{yr}^{-1}$. The accreting WD experiences two small He novae before transitioning to steady He shell-burning, leading to the growth of the $\mathrm{C} / \mathrm{O}$ core. The mass-transfer rate during this phase exceeds the stable burning rate (Brooks et al. 2016), implying mass-loss from the binary and an RL filling accretor. An intriguing possibility in this phase is the inhibition of direct impact accretion due to the accretor fully filling its RL. This may enhance the likelihood of stable mass-transfer for these systems. As more mass is transferred, the orbital period increases and the mass-transfer rate drops below the minimum steady burning rate and begins mild $\mathrm{He}$ flashes that are eventually strong enough to remove mass via RLOF in short ejection episodes. The last and largest of these flashes (Shen et al. 2010) involves only $10^{-2} M_{\odot}$ and unambiguously remains hydrostatic. After the last flash, the accreting WD quiescently grows to $1.04 M_{\odot}$, with a $0.94 M_{\odot} \mathrm{C} / \mathrm{O}$ core.

\section{Conclusions and Summary}

OW J0741 was discovered as one of bluest variable sources in the OmegaWhite survey. The VST observations revealed variability with a period of 22.6 minutes. Follow-up observations show that OW J0741 is an ultracompact sdOB binary with a compact companion with $P_{\text {orb }}=44.66279 \pm$ $1.16 \times 10^{-4}$ minutes, making it the most compact hot subdwarf binary known today.

High-S/N photometry obtained with Gemini exclude an eclipse that allows us to put tight constraints on the system parameters. Combining Gemini, Keck, and ULTRACAM light curves with spectroscopy, we find a mass ratio $q=M_{\mathrm{sdOB}} / M_{\mathrm{WD}}=0.32 \pm$ 0.10 , a mass for the sdOB $M_{\mathrm{sdOB}}=0.23 \pm 0.12 M_{\odot}$ and a WD companion mass $M_{\mathrm{WD}}=0.72 \pm 0.17 M_{\odot}$. The derived sdOB mass is inconsistent with the canonical mass for hot subdwarfs of $\approx 0.47 M_{\odot}$ and therefore the sdOB has not evolved from the standard hot subdwarf channel where the envelope of the subdwarf progenitor gets stripped at the tip of the red-giant branch.

To put constraints on the nature of the sdOB star, we compared the derived $T_{\text {eff }}$ and $\log g$ to evolutionary tracks for He-stars and He white dwarfs computed with MESA. For the He-star scenario, only a He shell-burning star with a mass around $0.462 M_{\odot}$ is consistent with the derived $T_{\text {eff }}$ and $\log g$. However, such a high mass is inconsistent with the derived 
Table 2

Results From the Individual Light Curve Fits

\begin{tabular}{|c|c|c|c|c|c|c|c|}
\hline Light Curve & $q=\frac{M_{\mathrm{sdOB}}}{M_{\mathrm{WD}}}$ & $\begin{array}{c}M_{\mathrm{sdOB}} \\
\left(M_{\odot}\right)\end{array}$ & $\begin{array}{c}R_{\text {sdOB }} \\
\left(R_{\odot}\right)\end{array}$ & $\begin{array}{l}M_{\mathrm{WD}} \\
\left(M_{\odot}\right)\end{array}$ & $\begin{array}{c}i \\
\left({ }^{\circ}\right)\end{array}$ & $\begin{array}{c}a \\
\left(R_{\odot}\right)\end{array}$ & $\frac{R_{\mathrm{sdOB}}}{a}$ \\
\hline Keck (2016 Nov 03) & $0.47 \pm 0.23$ & $0.40 \pm 0.25$ & $0.13 \pm 0.03$ & $0.84 \pm 0.22$ & $62.3 \pm 11.5$ & $0.45 \pm 0.04$ & $0.30 \pm 0.04$ \\
\hline Gemini (2016 Nov 28) & $0.29 \pm 0.13$ & $0.25 \pm 0.15$ & $0.12 \pm 0.02$ & $0.92 \pm 0.21$ & $50.1 \pm 6.6$ & $0.44 \pm 0.04$ & $0.27 \pm 0.03$ \\
\hline Gemini (2016 Dec 05) & $0.37 \pm 0.14$ & $0.32 \pm 0.18$ & $0.13 \pm 0.02$ & $0.91 \pm 0.22$ & $53.3 \pm 6.3$ & $0.45 \pm 0.04$ & $0.29 \pm 0.03$ \\
\hline Gemini (2016 Dec 06) & $0.39 \pm 0.16$ & $0.36 \pm 0.20$ & $0.13 \pm 0.02$ & $0.92 \pm 0.21$ & $55.0 \pm 6.1$ & $0.45 \pm 0.04$ & $0.29 \pm 0.03$ \\
\hline ULTRACAM $g^{\prime}$ (2016 Dec 07) & $0.56 \pm 0.25$ & $0.44 \pm 0.26$ & $0.14 \pm 0.03$ & $0.81 \pm 0.20$ & $69.6 \pm 11.4$ & $0.45 \pm 0.04$ & $0.31 \pm 0.03$ \\
\hline ULTRACAM r' (2016 Dec 07) & $0.50 \pm 0.26$ & $0.40 \pm 0.27$ & $0.14 \pm 0.03$ & $0.79 \pm 0.18$ & $64.4 \pm 12.8$ & $0.44 \pm 0.04$ & $0.31 \pm 0.03$ \\
\hline Combined Analysis & $0.32 \pm 0.10$ & $0.23 \pm 0.12$ & $0.11 \pm 0.02$ & $0.72 \pm 0.17$ & $57.4 \pm 4.7$ & $0.41 \pm 0.04$ & $0.28 \pm 0.02$ \\
\hline
\end{tabular}

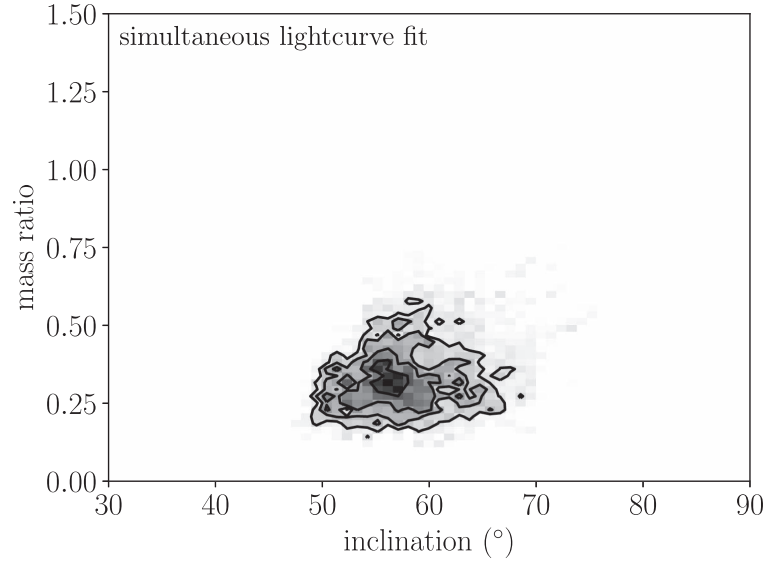

Figure 6. 2D Histogram of the results from the simultaneous light curve fit. The contours show the $1 \sigma, 2 \sigma$, and $3 \sigma$ confidence.

Table 3

Overview of the Derived Parameters for OW J0741

\begin{tabular}{llc}
\hline \hline Right Ascension & R.A. [hr] & $07: 41: 06.1$ \\
Declination & Decl. $\left.{ }^{\circ}\right]$ & $-29: 48: 11.0$ \\
Visual Magnitude & $m_{\mathrm{g}}$ & $20.02 \pm 0.11$ \\
\hline Atmospheric Parameters of the sdOB & \\
Effective Temperature & $T_{\text {eff }}[\mathrm{K}]$ & $39400 \pm 500$ \\
Surface Gravity & $\log g$ & $5.74 \pm 0.09$ \\
Helium Abundance & $\log y$ & $-0.14 \pm 0.06$ \\
\hline Orbital Parameters & & \\
& $T_{0}[\mathrm{MHJD}]$ & $57695.611284 \pm 1.66 \times 10^{-4}$ \\
Orbital Period & $P_{\mathrm{orb}}[\mathrm{min}]$ & $44.66279 \pm 1.16 \times 10^{-4}$ \\
RV Semi-amplitude & $K\left[\mathrm{~km} \mathrm{~s}^{-1}\right]$ & $422.5 \pm 21.5$ \\
System Velocity & $\gamma\left[\mathrm{km} \mathrm{s}{ }^{-1}\right]$ & $-14.0 \pm 11.5$ \\
Binary Mass Function & $f_{\mathrm{m}}\left[M_{\odot}\right]$ & $0.242 \pm 0.020$ \\
\hline Derived Parameters & & \\
Mass Ratio & $q=\frac{M_{\mathrm{sdOB}}}{M_{\mathrm{WD}}}$ & $0.32 \pm 0.10$ \\
sdOB Mass & $M_{\mathrm{sdOB}}\left[M_{\odot}\right]$ & $0.23 \pm 0.12$ \\
sdOB Radius & $R_{\mathrm{sdOB}}\left[R_{\odot}\right]$ & $0.11 \pm 0.02$ \\
WD Mass & $M_{\mathrm{WD}}\left[M_{\odot}\right]$ & $0.72 \pm 0.17$ \\
Orbital Inclination & $i\left[^{\circ}\right]$ & $57.4 \pm 4.7$ \\
Separation & $a\left[R_{\odot}\right]$ & $0.41 \pm 0.04$ \\
Distance & $d[\mathrm{kpc}]$ & $6.6 \pm 2.1$ \\
\hline & &
\end{tabular}

sdOB mass. For the white dwarf scenario, a He white dwarf with a mass of $0.320 M_{\odot}$ and a common envelope age of $\approx 1.1 \mathrm{Myr}$ is fully consistent with the derived system parameters. Although the evolutionary timescale is about 10

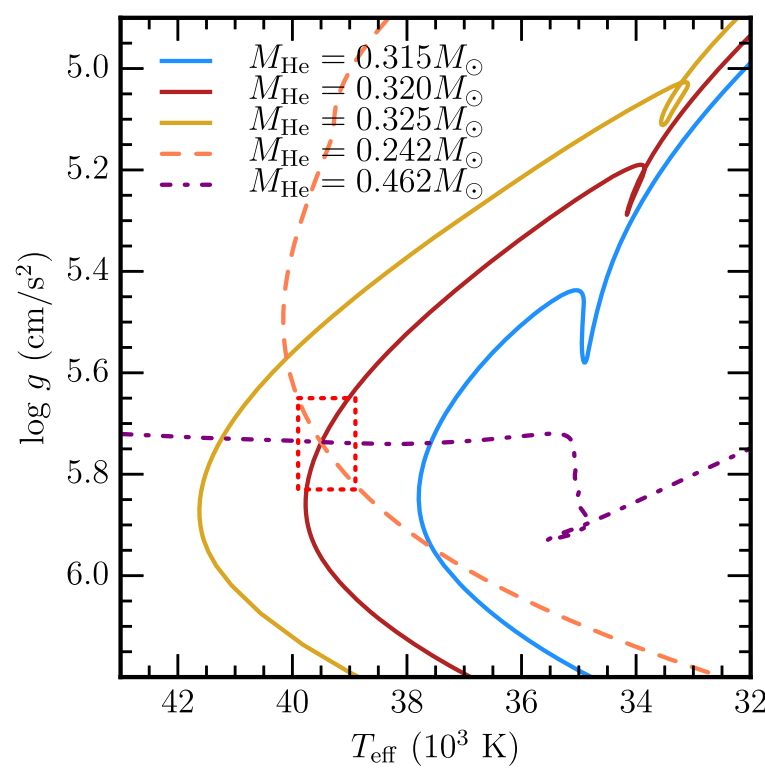

Figure 7. The red dotted line marks the observation box from the measurements and error bars given in Table 3. The three solid tracks are from He WD models of masses $0.315,0.320$, and $0.325 M_{\odot}$ (right to left), where the $0.320 M_{\odot}$ model spends $\approx 220,000$ years in the observation box and has a post-CE age of $\approx 1.1 \mathrm{Myr}$. The orange dashed curve is from the $0.242 \mathrm{M}_{\odot}$ model, which starts at the top of the plot, and spends $\approx 66,000$ years in the observation box and has a post-CE age of $\approx 11 \mathrm{Myr}$. The purple dot-dashed curve is from the $0.462 M_{\odot}$ model, which starts at the right of this plot and spends $\approx 4 \mathrm{Myr}$ in the observation box and has a post-CE age of $\approx 130 \mathrm{Myr}$.

times faster for a He white dwarf, we conclude that the most likely nature of the sdOB is a He white dwarf with a mass of $0.320 M_{\odot}$ and a common envelope age of $\approx 1.1 \mathrm{Myr}$.

To study the future evolution of the system we have constructed MESA models, assuming a $0.320 M_{\odot} \mathrm{He}$ white dwarf with a massive white dwarf companion of $0.85 M_{\odot}$, consistent with the derived mass ratio. In $17.6 \mathrm{Myr}$, the He WD will start RLOF at an orbital period of $\approx 5$ minutes. Depending on the spin-orbit synchronization timescale the object will either merge to form an $\mathrm{RCrB}$ star or end up as a stable accreting AMCVn-type system with a He WD donor. Better understandings of the spin-orbit synchronization timescales are required to decide whether the system will merge or prevent the merger. As an AMCVn, the system will show weak He shell flashes, but none strong enough to trigger a double-detonation SN Ia. Therefore, whether the system merges on contact or not, we conclude that this binary is not an SN Ia progenitor.

This work was supported by the GROWTH project funded by the National Science Foundation under grant No. 1545949. 
J.v.R. acknowledges support by the Netherlands Research School of Astronomy (NOVA) and the foundation for Fundamental Research on Matter (FOM). This research is funded in part by the Gordon and Betty Moore Foundation through Grant GBMF5076 and was also supported by the National Science Foundation under grant PHY 11-25915. We acknowledge stimulating workshops at Sky House where these ideas germinated.

T.R.M. acknowledge the support from the Science and Technology Facilities Council (STFC), ST/L00733. S.G. is supported by the Deutsche Forschungsgemeinschaft (DFG) through grant GE2506/9-1. D.K. acknowledges financial support from the National Research Foundation of South Africa. P.J.G. acknowledges support from NOVA for the original OmegaWhite observations and hospitality of the Kavli Institute for Theoretical Physics. Armagh Observatory is core funded by the Northern Ireland Executive through the Department for Communities.

This paper uses observations made at the South African Astronomical Observatory. Based on observations obtained at the European Southern Observatory, proposal 297.D-5010. Some results presented in this paper are based on observations obtained at the Gemini Observatory, proposal ID GS-2016B-FT-9, which is operated by the Association of Universities for Research in Astronomy, Inc., under a cooperative agreement with the NSF on behalf of the Gemini partnership: the National Science Foundation (United States), the National Research Council (Canada), CONICYT (Chile), Ministerio de Ciencia, Tecnología e Innovación Productiva (Argentina), and Ministério da Ciência, Tecnologia e Inovação (Brazil). The authors thank the staff at the ESO Paranal and Gemini South observatories for performing the observations in service mode. Some of the data presented herein were obtained at the W.M. Keck Observatory, which is operated as a scientific partnership among the California Institute of Technology, the University of California and the National Aeronautics and Space Administration. The Observatory was made possible by the generous financial support of the W.M. Keck Foundation. The authors wish to recognize and acknowledge the very significant cultural role and reverence that the summit of Maunakea has always had within the indigenous Hawaiian community. We are most fortunate to have the opportunity to conduct observations from this mountain. We thank the Swift team for the granting our DDT request.

Facilities: Gemini:South (GMOS-S), Keck:I (LRIS), VLT: Antu (FORS2), NTT (ULTRACAM), SALT (SALTICAM), Radcliffe (SHOC).

Software: LCURVE (Copperwheat et al. 2010), emcee (Foreman-Mackey et al. 2013), MESA (Paxton et al. 2011, 2013, 2015), FITSB2 (Napiwotzki et al. 2004).

\section{ORCID iDs}

T. Kupfer (iD https://orcid.org/0000-0002-6540-1484

T. R. Marsh (i) https://orcid.org/0000-0002-2498-7589

U. Heber (iD https://orcid.org/0000-0001-7798-6769

N. Blagorodnova (iD https://orcid.org/0000-0003-0901-1606

S. R. Kulkarni (i) https://orcid.org/0000-0001-5390-8563

R. Lunnan (ib https://orcid.org/0000-0001-9454-4639

T. A. Prince (iD https://orcid.org/0000-0002-8850-3627

\section{References}

Althaus, L. G., Miller Bertolami, M. M., \& Córsico, A. H. 2013, A\&A, 557, A19
Althaus, L. G., Serenelli, A. M., \& Benvenuto, O. G. 2001, MNRAS, 323, 471 Appenzeller, I., Fricke, K., Fürtig, W., et al. 1998, Msngr, 94, 1

Bauer, E. B., Schwab, J., \& Bildsten, L. 2017, arXiv:1707.05394

Bildsten, L., Shen, K. J., Weinberg, N. N., \& Nelemans, G. 2007, ApJL, 662, L95

Bloemen, S., Marsh, T. R., Østensen, R. H., et al. 2011, MNRAS, 410, 1787

Brooks, J., Bildsten, L., Marchant, P., \& Paxton, B. 2015, ApJ, 807, 74

Brooks, J., Bildsten, L., Schwab, J., \& Paxton, B. 2016, ApJ, 821, 28

Campbell, C. G. 1983, MNRAS, 205, 1031

Campbell, C. G. 1984, MNRAS, 207, 433

Claret, A. 2004, A\&A, 428, 1001

Coppejans, R., Gulbis, A. A. S., Kotze, M. M., et al. 2013, PASP, 125, 976

Copperwheat, C. M., Marsh, T. R., Dhillon, V. S., et al. 2010, MNRAS, 402, 1824

Dhillon, V. S., Marsh, T. R., Stevenson, M. J., et al. 2007, MNRAS, 378, 825

Fink, M., Röpke, F. K., Hillebrandt, W., et al. 2010, A\&A, 514, A53

Foreman-Mackey, D., Hogg, D. W., Lang, D., \& Goodman, J. 2013, PASP, 125,306

Fuller, J., \& Lai, D. 2014, MNRAS, 444, 3488

Geier, S., Fürst, F., Ziegerer, E., et al. 2015, Sci, 347, 1126

Geier, S., Hirsch, H., Tillich, A., et al. 2011, A\&A, 530, A28

Geier, S., Marsh, T. R., Wang, B., et al. 2013, A\&A, 554, A54

Green, G. M., Schlafly, E. F., Finkbeiner, D. P., et al. 2015, ApJ, 810, 25

Hamuy, M., Suntzeff, N. B., Heathcote, S. R., et al. 1994, PASP, 106, 566

Hamuy, M., Walker, A. R., Suntzeff, N. B., et al. 1992, PASP, 104, 533

Han, Z., Podsiadlowski, P., Maxted, P. F. L., \& Marsh, T. R. 2003, MNRAS, 341, 669

Han, Z., Podsiadlowski, P., Maxted, P. F. L., Marsh, T. R., \& Ivanova, N. 2002, MNRAS, 336, 449

Heber, U. 1986, A\&A, 155, 33

Heber, U. 2009, ARA\&A, 47, 211

Heber, U. 2016, PASP, 128, 082001

Hirsch, H. A., Heber, U., O'Toole, S. J., \& Bresolin, F. 2005, A\&A, 444, L61 Hook, I. M., Jørgensen, I., Allington-Smith, J. R., et al. 2004, PASP, 116, 425 Iben, I., Jr., \& Tutukov, A. V. 1991, ApJ, 370, 615

Jester, S., Schneider, D. P., Richards, G. T., et al. 2005, AJ, 130, 873

Kupfer, T., Geier, S., Heber, U., et al. 2015, A\&A, 576, A44

Kupfer, T., van Roestel, J., Brooks, J., et al. 2017, ApJ, 835, 131

Livne, E. 1990, ApJL, 354, L53

Livne, E., \& Arnett, D. 1995, ApJ, 452, 62

Macfarlane, S. A., Toma, R., Ramsay, G., et al. 2015, MNRAS, 454, 507

Macfarlane, S. A., Woudt, P. A., Dufour, P., et al. 2017a, MNRAS, 470, 732

Macfarlane, S. A., Woudt, P. A., Groot, P. J., et al. 2017b, MNRAS, 465, 434

Marsh, T. R., Nelemans, G., \& Steeghs, D. 2004, MNRAS, 350, 113

Maxted, P. f. L., Heber, U., Marsh, T. R., \& North, R. C. 2001, MNRAS, 326, 1391

Mereghetti, S., \& La Palombara, N. 2016, AdSpR, 58, 809

Napiwotzki, R., Karl, C. A., Lisker, T., et al. 2004, Ap\&SS, 291, 321

Nelemans, G. 2010, Ap\&SS, 329, 25

Nelemans, G., Portegies Zwart, S. F., Verbunt, F., \& Yungelson, L. R. 2001, A\&A, 368, 939

O'Donoghue, D., Buckley, D. A. H., Balona, L. A., et al. 2006, MNRAS, 372,151

Oke, J. B. 1990, AJ, 99, 1621

Oke, J. B., Cohen, J. G., Carr, M., et al. 1995, PASP, 107, 375

Patat, F., Moehler, S., O'Brien, K., et al. 2011, A\&A, 527, A91

Paxton, B., Bildsten, L., Dotter, A., et al. 2011, ApJS, 192, 3

Paxton, B., Cantiello, M., Arras, P., et al. 2013, ApJS, 208, 4

Paxton, B., Marchant, P., Schwab, J., et al. 2015, ApJS, 220, 15

Piersanti, L., Tornambé, A., \& Yungelson, L. R. 2014, MNRAS, 445, 3239

Ramspeck, M., Heber, U., \& Edelmann, H. 2001, A\&A, 379, 235

Savonije, G. J., de Kool, M., \& van den Heuvel, E. P. J. 1986, A\&A, 155, 51

Shen, K. J., \& Bildsten, L. 2014, ApJ, 785, 61

Shen, K. J., Kasen, D., Weinberg, N. N., Bildsten, L., \& Scannapieco, E. 2010, ApJ, 715, 767

Stroeer, A., Heber, U., Lisker, T., et al. 2007, A\&A, 462, 269

Toma, R., Ramsay, G., Macfarlane, S., et al. 2016, MNRAS, 463, 1099

Tutukov, A. V., \& Fedorova, A. V. 1989, SvA, 33, 606

Tutukov, A. V., \& Yungelson, L. R. 1990, SvA, 34, 57

Vennes, S., Kawka, A., O’Toole, S. J., Németh, P., \& Burton, D. 2012, ApJL, 759, L25

Verbunt, F., \& Rappaport, S. 1988, ApJ, 332, 193

Wang, B., Justham, S., \& Han, Z. 2013, A\&A, 559, A94

Woosley, S. E., \& Kasen, D. 2011, ApJ, 734, 38

Yungelson, L. R. 2008, AstL, 34, 620 